\title{
Index to Authors in Volume 10
}

Abdulla, Mahmood Ameen, 325

Abdullah, Noorlidah, 325

Ali, Abdul Manaf, 115

Ali, Hapipah Mohd, 325

Avtonomova, Anastasia V., 25

Badalyan, Susanna M., 155

Belitskii, Igor V., 25

Brčeski, Ilija, 351

Buchalo, Asja S., 369

Bukhman, Vladimir M., 25

Buswell, John A., 195

Caramori, Carlos A., 189

Cavas, Levent, 269

Chang, Shu-Ting, 195

Chang, Sue-Joan, 385

Chen, Ker-Shaw, 385

Cheung, Peter C. K., 255, 303

Choong, Yew Keong, 115

Cleaver, Matt, 219

Davitashvili, Elene, 171

Di Bernardi, Raffaello, 37

Dombo, Munehiko, 331

Du, Ming, 337

Duletić-Laušević, Sonja, 351

Eira, Augusto F., 189

Elgorashi, Esameldin E., 49

Elisashvili, Vladimir, 79, 171, 361

Ersel, Fadime Yilmaz, 269

Fernandes, Luiz Cláudio, 37

Figueiredo, Bonald Cavalcante, 37

Filho, José Hermênio Cavalcante

Lima, 37

Gharibyan, Narine G., 155

Ghods, Shirin, 149

Glamočlija, Jasmina, 351

Grayer, Renee J., 265

Gutiérrez-Lecuona, M. Teresa, 73

Guzmán, Gastón, 209

Hadar, Yitzhak, 79, 361

Harada, Toshie, 101

Hashimoto, Mamiko, 331

Hayashi, Kanako, 331

Hejaroude, Ghorban-Ali, 149

Helsper, Johannes P. F. G., 1

Ho, Wai-Jane, 181

Holliday, John, 219

Hsieh, Shu-Ling, 385
Hu, Shu-Hui, 385

$\mathrm{Hu}$, Xiaosong, 337

Huang, Shih-Jeng, 127, 245

Isakova, Elena B., 25

Isikhuemhen, Omoanghe S., 155

Janardhanan, Kainor K., 139

Job, Daniel, 93

Kachlishvili, Eva, 171

Kanojiya, Aarti, 163

Kapanadze, Ekaterine, 171

Katsy, Elena I., 65

Kawagishi, Hirokazu, 331

Keypour, Somayeh, 345

Khardziani, Tamar, 171

Kimura, Takashi, 331

Kodani, Shinya, 331

Krasnopolskaya, Larissa M., 25

Kumar, Satish, 87

Lai, Connie K. M., 255

Leifa, Fan, 37

Leontieva, Maria I., 25

Liang, Chih-Hung, 181

Liang, Zeng-Chin, 181, 279, 385

Lo, Sheng-Hua, 245

Maekawa, Nitaro, 49

Martins, Otávio A., 189

Mathew, John, 139

Mau, Jeng-Leun, 127, 245, 279

Meira, Dirceu R., 189

Menezes, Milena C., 189

Minato, Ken-ichiro, 235

Mishra, K. K., 379

Mitchell, Kevin, 55

Mizuno, Masashi, 15

Mohamed, Suhaila, 115

Moradali, Mohammad-Fata, 149, 345

Moreno, Andréa N., 37

Morimoto, Takanobu, 15

Mostafavi, Hossein, 149

Mykhailova, Oksana B., 369

Negriyko, Anatoly M., 369

Nevo, Eviatar, 79, 361

Nikitina, Valentina E., 65

Noor, Suzita Mohd, 325

Noordin, Mohamed Mustapha, 115

Ofodile, Lauretta N., 265

Ohno, Naohito, 101
Ponomareva, Elena G., 65

Poyedinok, Natalie L., 369

Rafati, Hassan, 345

Rana, Inder S., 163

Rebolj, Katja, 293

Riahi, Hossein, 345

Rony, Kuttikkadan A., 139

Rubel, Rosália, 37

Sabaratnam, Vikineswary, 325

Salmones, Dulce, 73

Sandhu, Sardul S., 163

Santa, Herta Stutz Dalla, 37

Satoh, Hiroshi, 49

Schelud'ko, Andrei V., 65

Sepčić, Kristina, 293

Sharma, S. R., 87

Sharma, V. P., 87

Shcherba, Victor V., 369

Silva, Giovanni Faria, 189

Simmons, Monnique S. J., 265

Simonić, Jasmina, 351

Singh, R. P., 379

Smina, Thozhuthumparambil P., 139

Soccol, Carlos Ricardo, 37

Songulashvili, George G., 79, 361

Stajić, Mirjana, 351

Stepanova, Lada V., 65

Sudheesh, Narayana P., 139

Takagi, Michihiro, 15

Tauwhare, Stephen, 55

Tokuyama, Shinji, 331

Tong, Chow Chin, 115

Tsai, Shu-Yao, 127, 245

Uma, N. U., 265

Umar, Nor Aini B., 115

Usov, Anatoliy I., 25

Van Griensven, Leo J. L. D., 1, 315

Vukojević, Jelena, 351

Wang, Cong, 337

Wang, Jinn-Chyi, 181, 385

Wasser, Solomon P., 79, 97, 361

Wei, Song, 1, 315

Wong, Ka-Hing, 255, 303

Wong, Kah-Hui, 325

Wu, Chiu-Yeh, 181, 279, 385

Wu, Tsai-Ping, 127

Zhao, Guanghua, 337 


\section{Index to Subjects in Volume 10}

adenosine, 55, 219

aeration level, 351

Agaricus bisporus, 1, 127, 163, 315

Agaricus bitorquis, 163

Agaricus brasiliensis, 1, 15, 181, 189, 315

agro wastes, 385

agro-wastes, 379

alternative substrates, 73

antagonistic activity, 155

antibacterial activity, 163, 345

antifungal effect, 155

anti-inflammatory, 49

antimicrobial activity, 265

antimycotic action, 155

antioxidant, 139, 315

antioxidant activity, 127, 245

antioxidant components, 127, 245

antioxidant enzymes, 269

antiproliferation, 255

antitumor, 37, 139

antitumor activity, 25

Antrodia camphorata, 315

apoptosis, 115

applied mushroom biology, 195

artist conk mushroom, 149

atopic dermatitis, 15

Auricularia polytricha, 315

$\beta$-glucan, 1, 101, 315

Bacillus subtilis, 163, 265

bacterial O-specific

polysaccharide, 65

Basidiomycetes, 65

biochemical composition, 303

biocontrol agent, 155

bioconversion, 385

biological efficiency, 87, 379, 385

biomass yield, 25

biopharmacological effects, 303

biosynthetic activity, 369

cancer, 255

carbohydrate specificity, 171

carbon source, 351

catalase, 269

chalcone, 331

characterization, 361

chelating ability, 127, 245

chemical composition, 189

chloroform extract, 345

chongcao, 219

co-cultivation, 65 coherence, 369

Coprinus comatus, 1, 315

cordycepic acid, 219

cordycepin, 219

Cordyceps, 219

Cordyceps robertsii, 55

Cordyceps sinensis, 55, 219

Cordyceps sp., 315

culinary-medicinal mushrooms, 65, 87, 181, 189, 235, 245, 279, 303, 385

cultivation technology, 379

cultivation, 181, 303

cultured mycelium, 139

cyclooxygenase-1, 49

cyclooxygenase-2, 49

cytoprotection, 325

DBA/2, 101

dectin-1, 1, 101

deoxynucleosides, 219

distribution, 209

dongchongxiacao, 219

edible mushrooms, 127, 195

enzyme kinetic, 361

ethnomycology, 209

exopolysaccharide, 279

Flammulina velutipes, 87

fruiting body, 181

fungal antibiotic, 163

Ganoderma applanatum, 149

Ganoderma lucidum, 1, 37, 79, 315, 345, 351, 361, 369, 379

Ganoderma, 115

gastric ulcer, 325

genetic crosses, 73

glucose, 79

glutathione peroxidase, 269

GM-CSF, 101

Grifola frondosa, 65, 315

growth, 369

heavy metal, 181

Hericium erinaceus, 315, 325, 385

Hericium laciniatum, 385

higher Basidiomycetes, 171

high-performance liquid

chromatography, 55

histology, 325

hydroxyethyladenosine, 219
Hypsizygus marmareus, 25

Hypsizygus ulmarius, 25

$\mathrm{IC}_{50}, 115$

immunoglobulin E (IgE), 15

immunomodulating activities, 101

immunomodulating effect, 235

immunomodulator, 37, 219

immunomodulatory effects, 1

in vitro, 337

India, 379

innate immunity, 1

inoculum density, 351

interferon (IFN)- $\gamma, 15$

interleukin (IL)-4, 15

interleukins, 337

King Tuber Oyster Mushroom, 303

laccase, 79, 361

Lactobacillus lactis, 163

lectin activity, 171

lectin-carbohydrate biospecific interactions, 65

Lentinus boryanus, 73

Lentinus edodes, 315

leukemic cells, 255

light irradiation, 369

lignocellulolytic enzymes, 87

lignocellulose, 171

lignocellulosic, 79

Ling Zhi mushroom, 37, 115

Ling Zhi or Reishi mushroom, 351, 361

lipid peroxidation, 269

lipid raft, 293

lymphocyte cells, 337

macroelements, 351

MDA-MB-435, 115

medicinal and edible mushrooms, 49

medicinal mushrooms, 1, 25, 37, 55, 79, 101, 115, 127, 139, 149, 155, 163, 171, 195, 209, 255, 265, 293, 315, 325, 331, 337, 345, 351, 361, 369, 379

Mexico, 73, 209

microelements, 351

micromorphology, 369

MnP, 79

MRSA, 331

MS, 331

murine macrophage, 235 
mushroom-based products, 155 mushroom biotechnology, 195 mushroom cultivation, 73 mushroom fruiting, 293 mushroom mycorestoration, 195 mushroom organizations, 195 mushroom science, 195 mycelia free culture filtrate, 163 mycelial biomass, 279 mycelium, 115

natural killer cells, 337

NC/Nga mice, 15

necrosis, 115

nitric oxide, 235

nitrogen source, 351

NK cells, 337

NMR, 331

nucleoside, 55

nutritive value, 189

ostreolysin, 293

oyster mushroom, 293

Phellinus linteus, 1, 315

phenolic compounds, 265

Pleurotus citrinopileatus, 235, 279

Pleurotus ostreatus, 293

Pleurotus tuberregium, 155, 303

polyphenols, 1, 315

Polyporus rhinocerus, 255

polysaccharides, 1, 25, 219, 255, 369

pore-forming protein, 293

pro-oxidant, 315

Pseudomonas syringae, 265

purification, 361

RAW264, 235

reducing power, 127, 245

Reishi mushroom, 37

Reishi or Ling Zhi mushroom, 345

relationships, 209

response surface methodology, 279

ROS, 1, 315

Royal Sun Agaricus, 181

saturated fatty acid, 149

scavenging ability, 127, 245

SCG, 101

sclerotium, 255, 303

screening, 171

Se-enriched Ganoderma

lucidum, 337 selenium-containing protein, 337

solid-state culture, 37

Sparassis crispa, 331

submerged and solid-state

fermentation, 171

submerged cultivation, 25

submerged culture, 279

sugar hapten, 65

superoxide dismutase, 269

supplements, 87

Th1/Th2 balance, 15

thin layer chromatography, 149

TNF- $\alpha, 235$

toxicity, 293

Traditional Chinese Medicine, 337

traditions, 209

Trametes versicolor, 315

Trametes, 265

tumor necrosis factor- $\alpha, 101$

tumor, 219

Volvariella volvacea, 139

wild edible Agaricales

mushrooms, 269

xylose, 79

yield, 87, 385 


\section{Reviewers for Volume 10}

S. M. Badalyan (Armenia)

R. Beelman (USA)

A. S. Buchalo (Ukraine)

J. A. Buswell (P. R. of China)

S. T. Chang (Australia)

P. C. K. Cheung (Hong Kong, SAR of P. R. China)

M. Ya. Didukh (Ukraine)

N. P. Denisova (Russia)

V. I. Elisashvili (Georgia)

L. J. L. D. van Griensven (The Netherlands)

G. Guzmán (Mexico)

X-G. He (USA)

Ch. Hobbs (USA)

J. Holliday (USA)

T. Ikekawa (Japan)

O. S. Isikhuemhen (USA)

D. Job (Switzerland)

H. Kawagishi (Japan)

T. Kiho (Japan)

B. K. Kim (South Korea)

C. N. Lin (P. R. of China)

H.-C.P. Lo (Taiwan, Rep. of China)

J. Mahajna (Israel)

G. Mata (Mexico)
J-L. Mau (Taiwan, Rep. of China)

N. Mikiashvili (Georgia)

M. Mizuno (Japan)

E. Nevo (Israel)

M.-L. Ng (Singapore)

T. B. Ng (Hong Kong, SAR of P. R. China)

N. Ohno (Japan)

R. Petrova (Bulgaria)

R. Poder (Austria)

M. Rai (India)

N. J. Rowan (UK)

D. J. Royse (USA)

V. Šašek (Czech Republic)

J. E. Smith (UK)

E. F. Solomko (Ukraine)

M. Stajić (Serbia)

P. Stamets (USA)

T. Stijve (Switzerland)

P. A. Volz (USA)

G. Zervakis (Greece)

Sh. Zhou (Singapore)

C. Zhuang (USA)

I. Zmitrovich (Russia) 